\title{
Deformation dependence of nuclear clusterization
}

\author{
J. Cseh* and A. Algora \\ Institute of Nuclear Research of the Hungarian Academy of Sciences, Debrecen, Pf. 51, Hungary-4001 \\ J. Darai \\ Institute of Experimental Physics, University of Debrecen, Debrecen, Bem tér 18/A, Hungary-4026 \\ P. O. Hess \\ Instituto de Ciencias Nucleares, UNAM, Circuito Exterior, C.U., A.P. 70-543, 04510 México, D.F., Mexico
}

(Received 3 June 2004; published 28 September 2004)

\begin{abstract}
The allowed and forbidden binary cluster configurations of the ground, superdeformed, and hyperdeformed states of some nuclei are determined, based on the (real or effective) U(3) selection rule. The stability of the cluster configurations from the viewpoint of the binding energy is also investigated.
\end{abstract}

DOI: 10.1103/PhysRevC.70.034311 PACS number(s): 21.60.Fw, 21.60.Gx, 27.30.+t, 27.90.+b

\section{INTRODUCTION}

Clusterization is an important effect in atomic nuclei. It may show up in light nuclei, as well as in heavy ones, near the ground state, as well as in highly excited regions, in states of spherical as well as of deformed shapes, at low and high spins, etc. [1]. The clusters also have a large variety; they can contain only a few nucleons, like, e.g., $\alpha$ particle, but there can be heavier ones as well, like, e.g., some C, O, $\mathrm{Mg}$, or $\mathrm{Si}$ isotopes, and in light of the recent experiments on cold spontaneous fission, they may even contain as many nucleons as 108 [2]. Therefore the question of what cluster configuration is allowed in a particular nuclear state is of utmost interest, and has been approached from different angles.

The binding energy of a (possible) cluster configuration is obviously an important factor. It is essential not only from the viewpoint of the penetrability of a cluster decay [3], but also from the viewpoint of the stability of the cluster structure. This aspect of clusterization has recently been studied extensively by Buck and co-workers [4,5], based on an empirical rule using the experimental data for binding energies.

Another important point is related to the microscopic structure, more specifically to the antisymmetrization of the total wave function of a nuclear state. In other words, one can say that the building up procedure of a nucleus from lighter clusters is governed by the energy-minimum principle and by the Pauli-exclusion principle. Thus it may very well happen that an energetically favored cluster configuration is not allowed due to the exclusion principle. The microscopic nature of clusterization can be approached either from the side of the parent nucleus, describing it with a shell-model [6], Hartree-Fock [7], Nilsson-Strutinsky [8], deformed Woods-Saxon [9], etc., method, or from the cluster side [10].

The complexity of this problem can be visualized in geometrical terms: the clusters themselves can be deformed, and their orientation with respect to the molecular axis is free to

\footnotetext{
*Electronic address: cseh@atomki.hu
}

change. In addition, different cluster configurations may coexist; their wave functions are not orthogonal to each other (in a microscopic description).

The inclusion of all these features makes the detailed cluster studies rather involved, and in many cases, like for the large number of nucleons, or high excitation energies, even prohibitively complicated. Thus simplified methods, which are systematically applicable, but still contain the main ingredients of the microscopic studies, are highly desirable.

In this paper we apply a selection-rule technique for the determination of allowed binary cluster configurations of the ground, superdeformed, and hyperdeformed states of the ${ }^{36} \mathrm{Ar}$ and ${ }^{252} \mathrm{Cf}$ nuclei, in order to improve our understanding on the deformation dependence of nuclear clusterization. In addition to the yes-or-no statements of the selection rule, we characterize the forbiddenness quantitatively. This consideration is based on the microscopic structure of the states in question, therefore it gives rise to a deformation dependence.

A comparison with the result of the maximal stability criterium [4], based on the binding energies, is also given. This latter quantity depends only on the parent nucleus and the clusters, but not on the specific state of the parent nucleus.

The structure of this paper is as follows. In Sec. II we describe the methods which are applied in searching for possible clusterizations, then the ${ }^{36} \mathrm{Ar}$ and ${ }^{252} \mathrm{Cf}$ cases are presented in Sec. III and IV, and finally some conclusions are drawn.

\section{SELECTION OF CLUSTERIZATION}

The structural selection rule we apply is based on the $\mathrm{U}(3)$ symmetry, what is known to be a good approximate symmetry of light nuclei [11], and its role in the clusterization was also observed in early studies [12], followed by the understanding of its importance from different aspects of clusterization [13]. The simple $\mathrm{U}(3)$ selection rule reads

$$
\left[n_{1}, n_{2}, n_{3}\right]=\left[n_{1}^{(1)}, n_{2}^{(1)}, n_{3}^{(1)}\right] \otimes\left[n_{1}^{(2)}, n_{2}^{(2)}, n_{3}^{(2)}\right] \otimes\left[n^{(R)}, 0,0\right],
$$

where $\left[n_{1}, n_{2}, n_{3}\right]$ is the set of (approximate) $\mathrm{U}(3)$ quantum numbers of the parent nucleus, the superscript $(i)$ stands for 
the $i$ th cluster, and $(R)$ indicates relative motion.

In medium and heavy nuclei, however, the U(3) symmetry is not valid in its original form, due to the importance of the symmetry-breaking interactions, like spin orbit and pairing. Nevertheless, it was found [14] that in spite of the strong symmetry-breaking interactions a generalized U(3) symmetry, called effective or quasidynamical U(3) symmetry, may survive even for heavy nuclei. It is very general in the sense that the energy eigenfunctions are composed as linear combinations of basis states belonging to different $\mathrm{U}(3)$ irreducible representations (irreps). In other words, the symmetry breaking is much stronger than that of the dynamical breaking known from many algebraic models like, e.g., the interacting boson model [15] or the semimicroscopic algebraic cluster model [16]. In these latter cases the symmetrybreaking interactions only split the symmetric basis states, but do not mix them, due to the fact that the Hamiltonian is expressed in terms of the invariant operators of an algebra chain. In the case of the effective symmetry, however, the symmetric basis states are not only split, but even mixed with each other. Nevertheless, the mixing is a special one, it is a coherent mixing of different irreducible representations, which results in an approximate $\mathrm{U}(3)$ symmetry. This symmetry is determined by effective or average $U(3)$ quantum numbers, which represent a coherent superposition of many basis states of the real U(3) symmetry. The physical condition for the special mathematical structure of the effective symmetry is the adiabatic decoupling of the single nucleon motion and the collective motion of the nucleus. In Ref. [17] a method is developed for the determination of the effective U(3) quantum numbers of the heavy nuclei, based on the occupation of the asymptotic Nilsson orbits. The procedure, which was originally invented for the large prolate deformation, was extended in Ref. [18] for the oblate shape and small deformations as well, based on the expansion of singleparticle orbitals in terms of asymptotic Nilsson states.

When applying the effective $\mathrm{U}(3)$ symmetry in cluster studies, it describes an average (or effective) clusterization. Contrary to the average clusterization of the phenomenological approach [4] this indicates a quantum mechanical superposition, as discussed in the previous paragraph, not a statistical average. Furthermore, one has to keep in mind that the different cluster configurations are not orthogonal to each other, therefore, just like for the real $\mathrm{U}(3)$ symmetry of the light nuclei, different effective cluster configurations may have a large overlap. The concept of effective symmetry is applicable also to light nuclei, and when the simple leading representation approximation is valid, the real and effective U(3) quantum numbers usually coincide [18]. This circumstance gives a straightforward way for the extension of the simple selection rule consideration.

When a cluster configuration is forbidden, we can characterize its forbiddenness quantitatively in the following way [19]. The distance between a $\mathrm{U}(3)$ reaction channel and the irrep of the parent nucleus is defined as: $\min \left[\sqrt{\left(\Delta n_{1}\right)^{2}+\left(\Delta n_{2}\right)^{2}+\left(\Delta n_{3}\right)^{2}}\right]$, where $\Delta n_{i}=\left|n_{i}-n_{i, k}^{c}\right|$. Here $n_{i}$ refers to the $\mathrm{U}(3)$ representation of the parent nucleus, while $n_{i, k}^{c}$ stands for the $\mathrm{U}(3)$ representation of channel $c$, obtained from the right-hand side of Eq. (1), with the $k$ index distin- guishing the different product representations. Based on this quantity we determine, for reasons of convenience, the reciprocal forbiddenness $S$ in such a way that $0 \leqslant S \leqslant 1$ :

$$
S=\frac{1}{1+\min \left[\sqrt{\left(\Delta n_{1}\right)^{2}+\left(\Delta n_{2}\right)^{2}+\left(\Delta n_{3}\right)^{2}}\right]} .
$$

Then $S=0$ and $S=1$ correspond to completely forbidden and allowed clusterizations, respectively.

The criterium of maximal stability [4], which represents a complementer viewpoint for the selection of clusterization, requires the largest value of the summed differences of the measured binding energies and the corresponding liquid drop values:

$$
D(1,2)=\left[B(1)-B_{L}(1)\right]+\left[B(2)-B_{L}(2)\right],
$$

where $B(i)$ is the experimental binding energy of the $i$ th cluster [20], while $B_{L}(i)$ stands for liquid drop value [4].

In the generalized version of the method, as we apply it here, a further condition is also taken into account, which is called dipole constraint [4]. It is based on the observation that electric dipole transitions are very weak, therefore the decomposition $A_{T} \rightarrow A_{1}+A_{2}$ is expected to be close to satisfying the constraint

$$
\frac{Z_{1}}{A_{1}} \approx \frac{Z_{I}}{A_{I}} \approx \frac{Z_{2}}{A_{2}} .
$$

Thus when considering even-even nuclei for a fixed charge, e.g., $Z_{1}$, two mass values $A_{1}$ and $\left(A_{1}+2\right)$ approximate the dipole condition

$$
\frac{Z_{1}}{A_{1}} \geqslant \frac{Z_{T}}{A_{T}} \geqslant \frac{Z_{1}}{\left(A_{1}+2\right)},
$$

and a weighted average of them satisfies it exactly,

$$
\begin{gathered}
a\left(\frac{Z_{1}}{A_{1}}\right)+b\left(\frac{Z_{1}}{\left(A_{1}+2\right)}\right)=\left(\frac{Z_{T}}{A_{T}}\right), \\
a+b=1 .
\end{gathered}
$$

The mean value of the binding energy is then defined as

$$
\langle D(1,2)\rangle=\left.a D(1,2)\right|_{Z_{1}, A_{1}}+\left.b D(1,2)\right|_{Z_{1},\left(A_{1}+2\right)},
$$

where " $\left.\right|_{Z_{1}, A_{1}}$ " refers to the charge and mass values.

The criterium of maximal stability, as it is presented here, and discussed more in detail in Ref [4], is based exclusively on the binding energies together with the no-dipole constraint, and contains no further ingredients, like, e.g., intercluster potentials.

\section{III. ${ }^{36} \mathrm{Ar}$}

The reason why the ${ }^{36} \mathrm{Ar}$ nucleus is especially suited for the study of deformation dependence of clusterization is that its superdeformed state has recently been observed experimentally [21] and studied theoretically [21,22]. Furthermore, there is a theoretical prediction for its hyperdeformed state as well [23]. 
TABLE I. The quantum numbers of the ground, superdeformed, and hyperdeformed states of the ${ }^{36} \mathrm{Ar}$ nucleus.

\begin{tabular}{lcc}
\hline \hline State & $\hbar \omega$ & {$\left[n_{1}, n_{2}, n_{3}\right]$} \\
\hline Ground & 0 & {$[20,20,12]$} \\
Superd. (a) & 4 & {$[32,12,12]$} \\
Superd. (b) & 4 & {$[32,14,10]$} \\
Hyperd. (a) & 12 & {$[48,8,8]$} \\
Hyperd. (b) & 6 & {$[36,12,10]$} \\
\hline \hline
\end{tabular}

A highly deformed band was inferred in ${ }^{36} \mathrm{Ar}$ from the study of the Nilsson diagram at $\epsilon_{2} \sim 0.4$ [24], and the experimentally found rotational band with $\beta_{2} \approx 0.45$ (low-spin) quadrupole deformation and terminating with the $J^{\pi}=16^{+}$ state seems to correspond to this expectation. Furthermore, it can be interpreted both in cranked Nilsson-Strutinsky calculation [22], and in large-scale $s d-p f$ shell-model calculation [21] as a configuration of two protons and two neutrons moved to the $p f$ major shell. Therefore it is considered to be a superdeformed band.

In determining the $\mathrm{U}(3)$ symmetry of this band we have followed two different methods. On the one side one can apply elementary considerations as follows. The largest prolate deformation that can be obtained from four-nucleon excitation to the $p f$ major shell belongs to the $[32,12,12]$ representation. It is remarkable that the same representation corresponds to the $\beta_{2}=0.6$ deformation parameter, which is generally considered to characterize the superdeformed shape, via the relation between the $(\beta, \gamma)$ parameters and the $(\lambda, \mu) \mathrm{SU}(3)$ labels, if we assume $4 \hbar \omega$ excitation [25]. According to the simple self-consistency argument [26] it gives a ratio of main axes 5:3. This state is indicated in Table I as "Superd. (a)."

On the other hand, one can derive the effective or quasidynamical U(3) symmetry quantum numbers from the occupation of Nilsson orbits, corresponding to the $\beta_{2} \approx 0.45 \mathrm{de}-$ formation, as it was proposed in Ref. [17]. In this way one gets the $[32,14,10] \mathrm{U}(3)$ representation, which also corresponds to $4 \hbar \omega$ excitation (with ratio of axes $25: 16: 14$ ). This state is indicated in Table I as "Superd (b)."

As for the possible hyperdeformed state in ${ }^{36} \mathrm{Ar}$, it was predicted from cranked Bloch-Brink $\alpha$-cluster model calculation with ratio of major to minor axis 3:1 [23], and a correspondence to some heavy-ion resonances were conjuctioned. This state has a U(3) symmetry $[48,8,8]$, noted as "Hyperd. (a)" in Table I, and corresponds to $12 \hbar \omega$ excitation. (According to the simple self-consistency argument it gives a ratio of main axes $33: 13$.) The effective $\mathrm{U}(3)$ quantum numbers, determined from the Nilsson scheme for the $\beta_{2} \approx 0.86$ deformation, are $[36,12,10]$ ["Hyperd. (b)"], corresponding to merely $6 \hbar \omega$ excitation (and ratio of axes 27:15:14).

The differences in the $\mathrm{U}(3)$ quantum numbers of the super- and hyperdeformed states reflect the uncertainty of the applied theoretical methods (or simple arguments) for the prediction of these extremely deformed states. Not surprisingly the deviation is much smaller in the superdeformed state than in the hyperdeformed one.
TABLE II. The allowed and forbidden binary cluster configurations of the ground, superdeformed ( $a$ and $b$ ), and hyperdeformed (a) states of ${ }^{36} \mathrm{Ar}$, in terms of mass numbers (see Table III for further details). The clusters are supposed to be in their ground states. In the case of hyperdeformed $(b)$ state the clusterizations indicated $*$ by are for bidden.

\begin{tabular}{|c|c|c|}
\hline State & Allowed & Forbidden \\
\hline \multirow[t]{7}{*}{ Ground } & & $18+18$ \\
\hline & & $19+17$ \\
\hline & & $\begin{array}{c}\cdots \\
22+14\end{array}$ \\
\hline & & $23+13$ \\
\hline & $24+12$ & \\
\hline & $25+11$ & \\
\hline & $\ldots$ & \\
\hline \multirow[t]{13}{*}{ Superd. } & & $18+18$ \\
\hline & & $19+17$ \\
\hline & & $\ldots$ \\
\hline & & $22+14$ \\
\hline & & $23+13$ \\
\hline & $24+12$ & \\
\hline & $25+11$ & \\
\hline & $26+10$ & \\
\hline & $27+9$ & \\
\hline & $28(p)+8$ & \\
\hline & & $28(0)+8$ \\
\hline & & $29+7$ \\
\hline & & $\ldots$ \\
\hline \multirow[t]{14}{*}{ Hyperd. } & $18+18$ & \\
\hline & $19+17$ & \\
\hline & $20+16^{*}$ & \\
\hline & $21+15^{*}$ & \\
\hline & $22+14$ & \\
\hline & $23+13$ & \\
\hline & $24+12$ & \\
\hline & $25+11$ & \\
\hline & $26+10$ & \\
\hline & $27+9$ & \\
\hline & $28(p)+8$ & \\
\hline & & $28(0)+8$ \\
\hline & & $29+7$ \\
\hline & & $\ldots$ \\
\hline
\end{tabular}

For the reason of completeness let us note here that for the ground state the effective U(3) quantum numbers coincide with the $[20,20,12]$ leading representation [18], and the self-consistency argument gives a ratio of axes 19:15.

Starting from these U(3) symmetries the application of the selection rule provides us with the results of Table II. There the cluster configurations are abbreviated by their mass numbers the detailed list is shown in Table III. We have considered all the possible binary configurations containing stable nuclei as clusters, and some further cases when an 
TABLE III. The relevant binary cluster configurations of ${ }^{36} \mathrm{Ar}$, abbreviated by their mass numbers in Table II.

\begin{tabular}{|c|c|}
\hline$A_{1}+A_{2}$ & Nuclei \\
\hline $18+18$ & $\begin{array}{l}{ }_{9}^{18} \mathrm{~F}+{ }_{9}^{18} \mathrm{~F} \\
{ }_{8}^{18} \mathrm{O}+{ }_{10}^{18} \mathrm{Ne}\end{array}$ \\
\hline $19+17$ & $\begin{array}{l}{ }_{10}^{19} \mathrm{Ne}+{ }_{8}^{17} \mathrm{O} \\
{ }_{9}^{19} \mathrm{~F}+{ }_{9}^{17} \mathrm{~F}\end{array}$ \\
\hline $20+16$ & ${ }_{10}^{20} \mathrm{Ne}+{ }_{8}^{16} \mathrm{O}$ \\
\hline $21+15$ & $\begin{array}{l}{ }_{11}^{21} \mathrm{Na}+{ }_{7}^{15} \mathrm{~N} \\
{ }_{10}^{21} \mathrm{Ne}+{ }_{8}^{15} \mathrm{O}\end{array}$ \\
\hline $22+14$ & $\begin{array}{l}{ }_{12}^{22} \mathrm{Mg}+{ }_{6}^{14} \mathrm{C} \\
{ }_{22}^{22} \mathrm{Na}+{ }_{7}^{14} \mathrm{~N} \\
{ }_{11}^{22} \mathrm{Ne}+{ }_{8}^{14} \mathrm{O}\end{array}$ \\
\hline $23+13$ & $\begin{array}{l}{ }_{12}^{23} \mathrm{Mg}+{ }_{6}^{13} \mathrm{C} \\
{ }_{11}^{23} \mathrm{Na}+{ }_{7}^{13} \mathrm{~N}\end{array}$ \\
\hline $24+12$ & ${ }_{12}^{24} \mathrm{Mg}+{ }_{6}^{12} \mathrm{C}$ \\
\hline $25+11$ & $\begin{array}{l}{ }_{13}^{25} \mathrm{Al}+{ }_{5}^{11} \mathrm{~B} \\
{ }_{12}^{25} \mathrm{Mg}+{ }_{6}^{11} \mathrm{C}\end{array}$ \\
\hline $26+10$ & $\begin{array}{l}{ }_{14}^{26} \mathrm{Si}+{ }_{4}^{10} \mathrm{Be} \\
{ }^{26} \mathrm{Al}+{ }_{5}^{10} \mathrm{~B} \\
{ }_{12}^{26} \mathrm{Mg}+{ }_{6}^{10} \mathrm{C}\end{array}$ \\
\hline $27+9$ & $\begin{array}{l}{ }_{14}^{27} \mathrm{Si}+{ }_{4}^{9} \mathrm{Be} \\
{ }_{13} \mathrm{Al}+{ }_{5}^{9} \mathrm{~B}\end{array}$ \\
\hline $28(p)+8$ & ${ }_{14}^{28} \mathrm{Si}(p)+{ }_{4}^{8} \mathrm{Be}$ \\
\hline $28(o)+8$ & ${ }_{14}^{28} \mathrm{Si}(o)+{ }_{4}^{8} \mathrm{Be}$ \\
\hline $29+7$ & $\begin{array}{l}{ }_{15}^{29} \mathrm{P}+{ }_{3}^{7} \mathrm{Li} \\
{ }_{14}^{29} \mathrm{Si}+{ }_{4}^{7} \mathrm{Be}\end{array}$ \\
\hline $30+6$ & $\begin{array}{l}{ }_{16}^{30} \mathrm{~S}+{ }_{2}^{6} \mathrm{He} \\
{ }_{30}^{30} \mathrm{P}+{ }_{3}^{6} \mathrm{Li} \\
{ }_{15}^{30} \mathrm{Si}+{ }_{4}^{6} \mathrm{Be}\end{array}$ \\
\hline $31+5$ & $\begin{array}{l}{ }_{16}^{31} \mathrm{~S}+{ }_{2}^{5} \mathrm{He} \\
{ }_{15} 1{ }^{5}+{ }_{3}^{5} \mathrm{Li}\end{array}$ \\
\hline $32+4$ & ${ }_{16}^{32} \mathrm{~S}+{ }_{2}^{4} \mathrm{He}$ \\
\hline $33+3$ & $\begin{array}{l}{ }_{17}^{33} \mathrm{Cl}+{ }_{1}^{3} \mathrm{H} \\
{ }_{16}^{33} \mathrm{~S}+{ }_{2}^{3} \mathrm{He}\end{array}$ \\
\hline $34+2$ & ${ }_{17}^{34} \mathrm{Cl}+{ }_{1}^{2} \mathrm{H}$ \\
\hline $35+1$ & ${ }_{17}^{35} \mathrm{Cl}+{ }_{1}^{1} \mathrm{H}$ \\
\hline
\end{tabular}

unstable nucleus is considered to be important from the clusterization point of view, like, e.g., ${ }^{8} \mathrm{Be}$. All the clusters were supposed to be in their ground state, and a simple leading term approximation was used in determining their U(3) symmetry (which usually coincides with the effective symmetries for light nuclei [18]). As for the ${ }^{28} \mathrm{Si}$ nucleus, right in the center of the $s d$ shell, a largely deformed prolate and oblate shape is known to coexist in its ground state with slightly more contribution from the oblate shape [27]. For this nucleus we have considered both shapes with " $o(p)$ " standing for the oblate (prolate) configuration. The values of reciprocal forbiddenness are shown in Fig. 1.

As a detailed example let us consider here the composition of the ${ }^{36} \mathrm{Ar}$ nucleus from those of ${ }^{12} \mathrm{C}$ and ${ }^{24} \mathrm{Mg}$. The shell-model configuration of ${ }^{12} \mathrm{C}$ is $(0)^{4}(1)^{8}$, indicating four
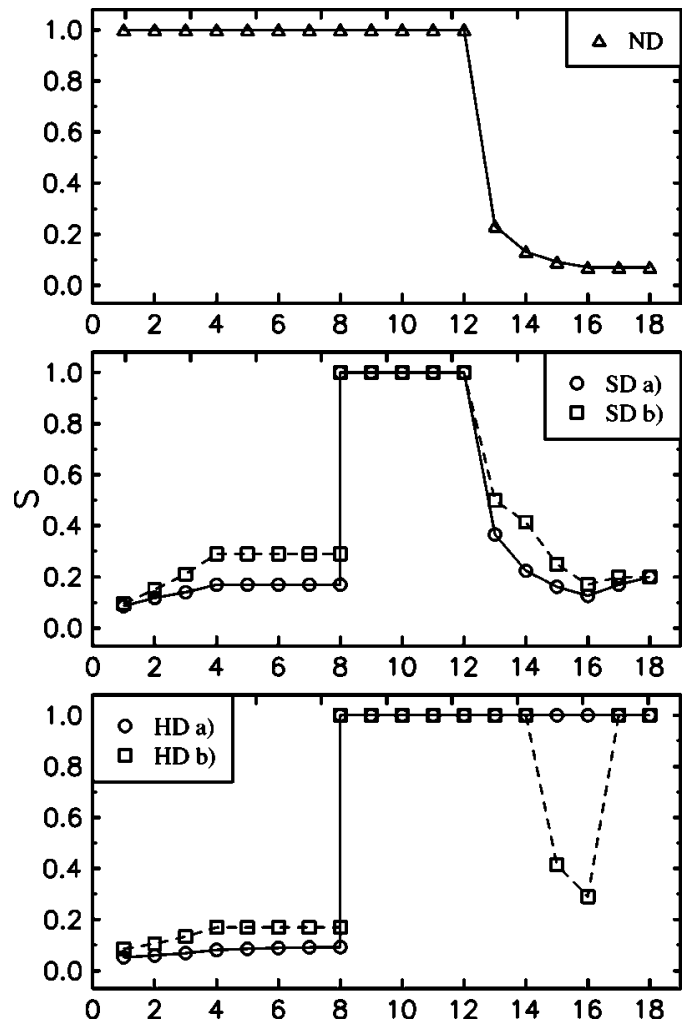

FIG. 1. Reciprocal forbiddenness for the ${ }^{36} \mathrm{Ar}$ clusterizations. ND stands for the normal deformed (ground) state, while SD and HD indicate super- and hyperdeformed states, respectively.

nucleons on the $0 \hbar \omega$ major shell (closed shell) and eight nucleons on the $1 \hbar \omega$ (valence) shell. For the other two nuclei the $1 \hbar \omega$ major shell is completely filled in: ${ }^{24} \mathrm{Mg}$ $(0)^{4}(1)^{12}(2)^{8},{ }^{36} \mathrm{Ar}(0)^{4}(1)^{12}(2)^{20}$.

The Pauli principle requires that when the two nuclei are amalgamated the nucleons of ${ }^{12} \mathrm{C}$ occupy empty singleparticle states above the closed $(0)^{4}(1)^{12}$ (i.e., ${ }^{16} \mathrm{O}$ ) core of ${ }^{24} \mathrm{Mg}$. Furthermore, the number of oscillator quanta can be increased only in one direction, along the molecular (e.g., z) axis (Harvey prescription) [28]. The rearrangement process can be illustrated most easily in the Cartesian basis: $\left(n_{x}, n_{y}, n_{z}\right)$, where $n_{i}$ shows the number of oscillator quanta along the $i$ axis in a single-nucleon state. Then the contents of the major shells are: $0 \hbar \omega:(0,0,0), 1 \hbar \omega:(0,0,1),(1,0,0)$, $(0,1,0), 2 \hbar \omega:(0,0,2),(1,0,1),(0,1,1),(2,0,0),(1,1,0),(0,2,0)$.

The detailed shell-model configuration of ${ }^{12} \mathrm{C}$ in this basis is $(0)^{4}\left\{(0,0,0)^{4}\right\}(1)^{8}\left\{(1,0,0)^{4}(0,1,0)^{4}\right\}$, giving rise to a leading $\mathrm{U}(3)$ representation of $[4,4,0]$. [The occupation of the $(0,0,1)$ single-particle state, due to the rearrangament $(0,0,1) \rightarrow(0,0,3)$ would result in a major shell excitation of ${ }^{36} \mathrm{Ar}$, which is not allowed in the ground state.] Similarly for ${ }^{24} \mathrm{Mg}: \quad(0)^{4}\left\{(0,0,0)^{4}\right\}(1)^{12}\left\{(1,0,0)^{4}(0,1,0)^{4}(0,0,1)^{4}\right\}$ $(2)^{8}\left\{(2,0,0)^{4}(1,1,0)^{4}\right\},[16,8,4]$, and for ${ }^{36} \mathrm{Ar}:(0)^{4}$ $\left\{(0,0,0)^{4}\right\}(1)^{12}\left\{(1,0,0)^{4}(0,1,0)^{4}(0,0,1)^{4}\right\} \quad(2)^{20}\left\{(2,0,0)^{4}\right.$ $\left.(1,1,0)^{4}(1,0,1)^{4}(0,1,1)^{4}(0,0,2)^{4}\right\},[20,20,12]$. The rearrangement of the 12 nucleons of ${ }^{12} \mathrm{C}$ is described by $\quad(0,0,0)^{4} \rightarrow(0,0,2)^{4},(1,0,0)^{4} \rightarrow(1,0,1)^{4},(0,1,0)^{4}$ $\rightarrow(0,1,1)^{4}$, corresponding to an increasement of the number of oscillator quanta by $2 \times 4+1 \times 4+1 \times 4=16$, which are 
carried by the relative motion $\left(n^{(R)}\right)$ in the cluster configuration (as the lowest value allowed by the Pauli principle). In terms of the $\mathrm{U}(3)$ selection rule it reads $[4,4,0] \otimes[16,8,4]$ $\otimes[16,0,0]=[20,20,12] \oplus \cdots$.

Once the molecular axis is fixed (in our example, $z$ ), then the shell-model configurations show the orientation of these deformed clusters as well. The oblate spheriodal ${ }^{12} \mathrm{C}$ nucleus has its symmetry (shortest) axis along the molecular (z) axis, and the triaxial ${ }^{24} \mathrm{Mg}$ lines up its shortest axis with $z$, the largest $(x)$ and the medium $(y)$ axes are perpendicular to it. (The other arrangement, longest $y$, medium $x$, would equally be possible.)

This example illustrates that the ground state of a nucleus requires a compact packing of a definite cluster configuration. Similar considerations show that excited states, like the superdeformed and hyperdeformed states, correspond to more stretched shapes of the same cluster configuration.

In light of this detailed discussion let us describe more specifically what we mean by saying that the clusters are supposed to be in their ground state. A nucleus is characterized by its $\mathrm{U}(3)$ symmetry quantum numbers, and a single $\mathrm{U}(3)$ irrep, that of its ground state, is taken into account, e.g., $[4,4,0]$ stands for ${ }^{12} \mathrm{C}$, and $[16,8,4]$ for ${ }^{24} \mathrm{Mg}$. However, a $\mathrm{U}(3)$ irrep may contain several states with different spin parity., e.g., the $[4,4,0] \mathrm{U}(3)$, i.e., $(0,4) \mathrm{SU}(3)$ irrep of ${ }^{12} \mathrm{C}$ contains a $K^{\pi}=0^{+}$rotational band with states $J^{\pi}=0^{+}, 2^{+}, 4^{+}$, while the $[16,8,4] \mathrm{U}(3)$, i.e., $(8,4) \mathrm{SU}(3)$ irrep of ${ }^{24} \mathrm{Mg}$ involves three rotational bands with $K^{\pi}=0^{+}\left(J^{\pi}=0^{+}, 2^{+}, 4^{+}\right.$, $\left.6^{+}, 8^{+}\right), \quad K^{\pi}=2^{+}\left(J^{\pi}=2^{+}, 3^{+}, 4^{+}, 5^{+}, 6^{+}, 7^{+}, 8^{+}, 9^{+}, 10^{+}\right), \quad K^{\pi}$ $=4^{+}\left(J^{\pi}=4^{+}, 5^{+}, 6^{+}, 7^{+}, 8^{+}, 9^{+}, 10^{+}, 11^{+}, 12^{+}\right)$. In other words, the intrinsic state of the cluster is well defined by its ground state, but collective rotations built on that are allowed. (In the language of the cluster models this scheme is called a strong [SU(3)] coupling scheme.)

An interesting general feature can be deduced from the results of Table II and III, and Fig. 1. In the ground state the asymmetric clusterizations are allowed and the symmetric ones are forbidden, while in the hyperdeformed state it is exactly the other way around. (The forbiddenness of the core-plus- $\alpha$-particle configuration may seem to be surprising, but one has to keep in mind that both clusters are supposed to be in their ground state, therefore in fact the ground-statelike ${ }^{32} \mathrm{~S}$ cluster is forbidden in the hyperdeformed state of ${ }^{36} \mathrm{Ar}$. The excitation of the core, or the inclusion of multicluster configuration, would allow the appearance of $\alpha$ cluster.) The superdeformed state shows an intermediate picture: both the most symmetric and the most asymmetric configurations are forbidden, but there is an island of allowed configuration in between.

It is remarkable that for the two different superdeformed symmetries (states) the allowed binary cluster configurations are exactly the same. In this case the small inconsistency, which can be found in the determination of the U(3) symmetry of the superdeformed shape based on different theoretical arguments, does not influence the possible clusterizations. For the hyperdeformed state one finds a deviation between the sets of allowed cluster configurations of the two (predicted) states $(a)$ and $(b)$, but even in this case the effect is much more reduced than in the primary values of the deformation parameters, or $\mathrm{U}(3)$ quantum numbers.

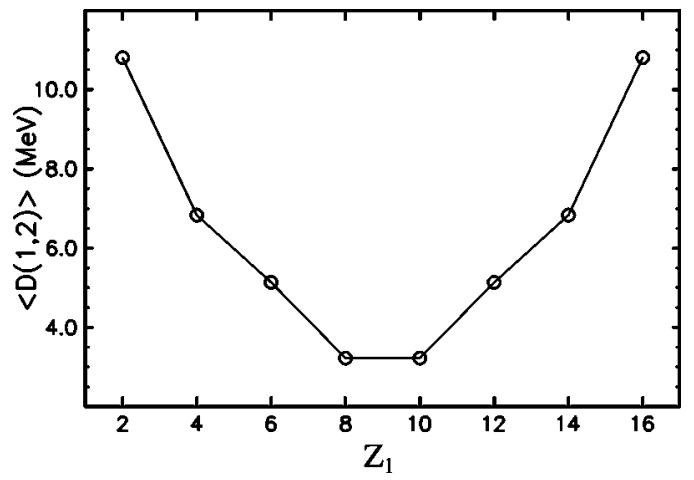

FIG. 2. Energetic stability of binary clusterizations in ${ }^{36} \mathrm{Ar}$.

The energetic stability of the different clusterizations is displayed in Fig. 2. Obviously it prefers core-plus-alpha configuration. ${ }^{8} \mathrm{Be}$ can also be interesting from this viewpoint, but it is probably more realistic to consider it in terms of a two-alpha configuration. ${ }^{12} \mathrm{C}$ is more probable than ${ }^{16} \mathrm{O}$. We have investigated the energetic stability of the possible clusterizations based on the modified prescription of Ref. [5] as well. It turned out that the figure of even-even cluster configurations did not change, while the configurations with odd- $A$ clusters are less favored.

It is interesting to note that there are a few cluster configurations which turn out to be allowed from the microscopic viewpoint both in the ground state, and in the superdeformed as well as in the hyperdeformed states. From among them the ${ }^{24} \mathrm{Mg}+{ }^{12} \mathrm{C}$ fragmentation contains $\alpha$-like nuclei, and is not so much suppressed by the energetic circumstances either. This means that each three states can be considered as a ${ }^{24} \mathrm{Mg}+{ }^{12} \mathrm{C}$ cluster configuration; the difference is made by the spatial arrangements of the clusters. In the ground state both the ${ }^{24} \mathrm{Mg}$ and the ${ }^{12} \mathrm{C}$ lines its shortest axis with the molecular axis $z$ (as mentioned beforehand); in the superdeformed state the ${ }^{24} \mathrm{Mg}$ can stand like in the ground state, but the ${ }^{12} \mathrm{C}$ turns its symmetry axis (the shortest axis) perpendicular to the molecular axis, while in the hyperdeformed state both cluster line their longest axis with $z$.

In Ref. [23] two sets of heavy-ion resonances were nominated for the hyperdeformed state: ${ }^{24} \mathrm{Mg}+{ }^{12} \mathrm{C}$ and ${ }^{20} \mathrm{Ne}$ $+{ }^{16} \mathrm{O}$. The first one turns out to be allowed not only in the hyperdeformed state $(a)$, predicted from $\alpha$-cluster model calculation, but also in the hyperdeformed $(b)$ state, obtained from the effective $\mathrm{U}(3)$ symmetry of the $\beta \approx 0.86$ deformation. Therefore the observation in this reaction channel cannot distinguish between the two candidates for the hyperdeformed state. The second one, however, is allowed only in state $(a)$, thus an observation in this channel would not be in line with the candidate $(b)$.

$$
\text { IV. }{ }^{252} \mathrm{Cf}
$$

Our interest in ${ }^{252} \mathrm{Cf}$ comes from recent experimental studies of the fission process using multidetector arrays that have revealed many interesting new features of this still puzzling phenomenon. Among these new results it is worth mentioning the first direct identification of neutronless binary 
TABLE IV. The quantum numbers of the ground [30], superdeformed, and hyperdeformed states of the ${ }^{252} \mathrm{Cf}$ nucleus.

\begin{tabular}{lcc}
\hline \hline State & $\beta_{2}$ & {$\left[n_{1}, n_{2}, n_{3}\right]$} \\
\hline Ground & 0.24 & {$[414,321,303]$} \\
Superd. & 0.60 & {$[520,285,267]$} \\
Hyperd. & 0.86 & {$[600,260,245]$} \\
\hline \hline
\end{tabular}

fission channels and the detection of exotic neutronless ternary processes in ${ }^{252} \mathrm{Cf}$ [2]. These new results and the more detailed spectroscopy in the second and third minima (of the energy surface) studied through fission [29] raise the question of to what extent microscopic effects play a role in the fission process.

In a simplified picture, fission can be viewed as a two step process: first, a cluster state is formed, and then the cluster state decays through the barrier. From this point of view a cluster basis can be considered as a natural choice. To study the microscopic aspects of this question, and in particular the effect of deformation, we have applied the U(3) selection rule to see if there is any microscopic preference for certain binary clusterizations of ${ }^{252} \mathrm{Cf}$ :

$$
{ }_{98}^{252} \mathrm{Cf} \rightarrow{ }_{Z}^{A} X+{ }_{98-Z}^{252-A} Y .
$$

The procedure applied here is based on the use of effective $\mathrm{U}(3)$ quantum numbers to characterize both the parent nucleus and the clusters. We have studied all possible binary clusterizations (308 fission channels) with $(8 \leqslant Z \leqslant 90)$. The starting point is always the deformation of the nucleus. Then we fill the Nilsson orbitals from below at that deformation value and determine the effective $(\lambda, \mu)$ using the relations of Refs. $[17,18]$. The parameters for the Nilsson Hamiltonian were taken from Ref. [22]. As an example we present in Table IV the U(3) effective quantum numbers determined for different deformations of ${ }^{252} \mathrm{Cf}$. The deformations used in our systematic study are taken from Ref. [30]. The use of theoretical values for the deformation instead of experimental ones is justified by the scope of our study: if we use only experimental values (see, for example Ref. [31]), many possible clusterization channels cannot be studied due to the lack of deformation values for one or both of the clusters. The number of quanta of the relative motion was determined using the Harvey prescription, as in the ${ }^{36} \mathrm{Ar}$ case. The preference of different cluster configurations is characterized by the reciprocal forbiddenness.

Using ground state deformations for the parent and daughter nuclei [30], all studied cluster configurations in question turn out to be forbidden (Fig. 3, upper part). A clear tendency towards cluster radioactivity (or very asymmetric fission) can be inferred from this figure. (We should remember that "forbidden" in the structural analysis might mean "suppressed.") One aspect that seems to be of particular interest: the low-lying cluster configuration of two prolate nuclei is not the pole-to-pole one, rather both clusters are inclined with respect to the molecular axis. For example, the lowest lying pole-pole configuration of ${ }^{144} \mathrm{Ba}+{ }^{108} \mathrm{Mo}$ lies at higher excitation than the one with inclined axes. This clus-
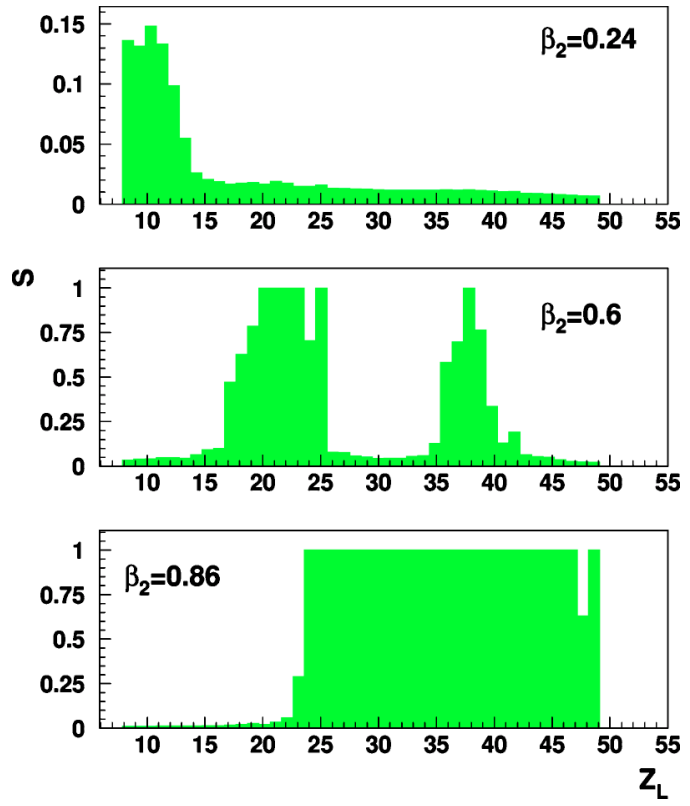

FIG. 3. (Color online) Reciprocal forbiddenness $S$ vs the $Z_{\text {light }}$ of the studied binary cluster configurations for the ${ }^{252} \mathrm{Cf}$ case. The values of $S$ correspond to mean values over channels that have the same $Z_{\text {light }}$ and different $A_{\text {light }}$.

terization is of particular interest because it has been seen in Ref. [2] as an example of a binary neutronless fission channel. This result shows that the pole-pole configurations, preferred by penetrability calculations, are highly Pauli forbidden. Therefore they can be only small components in the ground state wave function of ${ }^{252} \mathrm{Cf}$. The "compact packing" is favored by structure.

We have also addressed the question of whether there are allowed clusterizations in the case when we change the deformation of the parent nucleus to superdeformation $\left(\beta_{2}\right.$ $\sim 0.6)$ or hyperdeformation $\left(\beta_{2} \sim 0.86\right)$. The obtained results are presented in the lower parts of Fig. 3. As in the earlier calculations the clusters are considered to have ground state deformations. It is interesting to see that in these cases we have allowed clusterizations as well. In the case of a superdeformed ${ }^{252} \mathrm{Cf}$ the regions of allowed clusterizations correspond mainly to two particular regions in which: (a) both clusters have large prolate quadrupole deformation (region with $Z_{\text {light }} \sim 36$ ), (b) one cluster with prolate quadrupole deformation and the other with oblate deformation (region with $\left.Z_{\text {light }} \sim 22\right)$. For the hyperdeformed ${ }^{252} \mathrm{Cf}$ case more channels are open, and from Fig. 3 (lower panel) a clear tendency to symmetric clusterization can be inferred. The ${ }^{144} \mathrm{Ba}+{ }^{108} \mathrm{Mo}$ clusterization remains forbidden for the superdeformed case, but is allowed for the hyperdeformed case.

As in the ${ }^{36} \mathrm{Ar}$ case, we have also studied the criterium of maximum stability for comparison. The results of our study are presented in Fig. 4. It is clear from this figure that the preferred clusterizations of ${ }^{252} \mathrm{Cf}$, based on this approach, concentrate mainly in three regions around $Z=2, Z \sim 18$, and $Z \sim 50$. (The extension of the method according to Ref. [5] does not change the tendencies of even-even cluster configurations, while the ones with odd $A$ are less favored.) This result agrees with our $\mathrm{U}(3)$ procedure only for the $Z=2$ case 


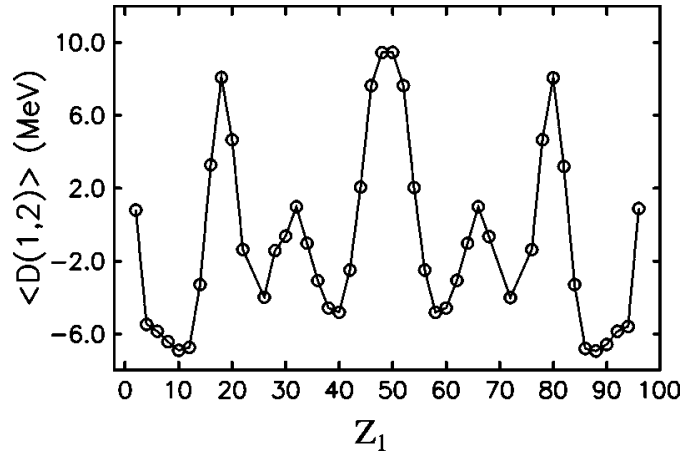

FIG. 4. Energetic stability of binary clusterizations in ${ }^{252} \mathrm{Cf}$.

[the $\alpha$ clusterization is allowed in the $\mathrm{U}(3)$ framework]. The other two regions are not favored in the U(3) approach if we consider ${ }^{252} \mathrm{Cf}$ in its ground state deformation (Fig. 3, upper panel), but $Z \sim 18$ is preferred if we assume ${ }^{252} \mathrm{Cf}$ in a superdeformed state and $Z \sim 50$ is allowed in the case of the hyperdeformed state.

\section{CONCLUSIONS}

In this paper we have studied the preference of binary clusterizations as a function of the deformation. A U(3) selection rule, based on the microscopic structure of the nuclei, and an empirical rule of maximal energetic stability was applied. The procedure was illustrated by the examples of two nuclei: ${ }^{36} \mathrm{Ar}$ and ${ }^{252} \mathrm{Cf}$.
The results clearly indicate that the two considerations, based on the microscopic structure on the one side and on the energetic stability on the other side, do not necessarily result in the same preference of cluster configurations. The structural selection rule has a similar tendency for the deformation dependence both in the case of the light ${ }^{36} \mathrm{Ar}$ nucleus and in the heavy ${ }^{252} \mathrm{Cf}$ nucleus. In the ground state the strongly asymmetric binary cluster configurations are preferred; in the hyperdeformed state the symmetric fragmentations are more likely, while in the superdeformed state the situation is in between, i.e., islands of allowed binary configurations appear. The most likely clusterizations are probably those which are in the overlapping regions of the preferences of the two complementary selecting procedures. In the ${ }^{36} \mathrm{Ar}$ case the ${ }^{24} \mathrm{Mg}+{ }^{12} \mathrm{C}$ configuration seems to be a remarkable one, because it is allowed from the microscopic viewpoint both in the ground, and in the superdeformed and hyperdeformed states, and it is only moderately suppressed from the energetic point of view. In ${ }^{252} \mathrm{Cf}$ the $Z_{\text {light }}=2,20$, and 48 regions are preferred in the ground, superdeformed, and hyperdeformed states, respectively.

Similar studies of other examples and of ternary (or multi) clusterizations could also be illuminative.

\section{ACKNOWLEDGMENTS}

This work was supported by the OTKA (Grant Nos. T37502 and T46791), the MTA-CONACyT joint project, and by DGAPA (Grant No. IN119002).
[1] Proceedings of the 8th International Conference on Clustering Aspects of Nuclear Structure and Dynamics, 2003, Nara, Japan (in press).

[2] J. H. Hamilton et al., J. Phys. G 20, L85 (1994); A. V. Ramaya et al., Phys. Rev. Lett. 81, 947 (1998).

[3] Nuclear Decay Modes, edited by D. N. Poenaru (IOP Publishing, Bristol, 1996).

[4] B. Buck, A. C. Merchant, and S. M. Perez, Few-Body Syst. 29, 53 (2000).

[5] B. Buck, A. C. Merchant, M. J. Horner, and S. M. Perez, Phys. Rev. C 61, 024314 (2000).

[6] I. Talmi, Simple Models of Complex Nuclei (Harwood Academic, Switzerland, 1993).

[7] S. Aberg, H. Flocard, and W. Nazarewitz, Annu. Rev. Nucl. Part. Sci. 40, 439 (1990).

[8] G. Leander and S. E. Larsson, Nucl. Phys. 239, 93 (1975).

[9] S. Aberg and L. O. Jönsson, Z. Phys. A 349, 205 (1994).

[10] A. Arima, H. Horiuchi, K. Kubodera, and Y. Takigawa, Adv. Nucl. Phys. 5, 345 (1972); K. Wildermuth and Y. C. Tang, A Unified Theory of the Nucleus (Academic, New York, 1977).

[11] J. P. Elliot, Proc. R. Soc. London, Ser. A 245, 128 (1958); 245, 562 (1958).

[12] B. F. Bayman and A. Bohr, Nucl. Phys. 9, 596 (1958/59).

[13] P. Kramer and M. Moshinsky, in Group Theory and its application, edited by E. M. Loebl (Academic, New York, 1968); V. G. Neudatschin, Yu. Smirnov, and N. F. Golovanova, Adv.
Nucl. Phys. 11, 1 (1979); K. T. Hecht, Nucl. Phys. 283, 223 (1977); J. Draayer, Nucl. Phys. 237, 157 (1975); Y. Suzuki, Nucl. Phys. 448, 395 (1986); K. Kato and H. Tanaka, Prog. Theor. Phys. 81, 841 (1989); J. Cseh, Phys. Rev. C 50, 2240 (1994).

[14] P. Rochford and D. J. Rowe, Phys. Lett. B 210, 5 (1988); D. J. Rowe, P. Rochford, and J. Repka, J. Math. Phys. 29, 572 (1988).

[15] F. Iachello and A. Arima, The Interacting Boson Model (Cambridge University Press, Cambridge, England, 1987).

[16] J. Cseh, Phys. Lett. B 281, 173 (1992); J. Cseh and G. Lévai, Ann. Phys. (N.Y.) 230, 165 (1994).

[17] M. Jarrio, J. L. Wood, and D. J. Rowe, Nucl. Phys. 528, 409 (1991).

[18] P. O. Hess, A. Algora, M. Hunyadi, and J. Cseh, Eur. Phys. J. A 15, 449 (2002).

[19] A. Algora and J. Cseh, J. Phys. G 22, L39 (1996).

[20] G. Audi and A. H. Wapstra, Nucl. Phys. 595, 409 (1995).

[21] C. E. Svensson, et al., Phys. Rev. Lett. 85, 2693 (2000); Phys. Rev. C 63, 061301(R) (2001).

[22] T. Bengtsson and I. Ragnarsson, Nucl. Phys. 436, 14 (1985); A. V. Afanasjev and I. Ragnarsson, Nucl. Phys. 591, 387 (1995).

[23] W. D. M. Rae and A. C. Merchant, Phys. Lett. B 279, 207 (1992). 
[24] R. K. Sheline, I. Ragnarsson, and S. G. Nilsson, Phys. Lett. 41, 115 (1972).

[25] D. J. Rowe, Rep. Prog. Phys. 48, 1419 (1985).

[26] A. Bohr and B. Mottelson, Nuclear Structure II (Benjamin, Reading, MA, 1975), p. 77.

[27] R. K. Sheline, S. Kubono, K. Morita, and M. H. Tanaka, Phys. Lett. 119, 263 (1982).

[28] M. Harvey, Proceedings of the 2nd Conference on Clustering Phenomena on Nuclei, College Park, 1975, USDERA Report
ORO-4856-26, p. 549.

[29] A. Krasznahorkay et al., Phys. Rev. Lett. 80, 2073 (1998); A. Krasznahorkay et al., Phys. Lett. B 461, 15 (1999); M. Hunyadi et al., Phys. Lett. B 505, 27 (2001).

[30] P. Möller, J. R. Nix, W. D. Myers, and W. J. Swiatetecki, At. Data Nucl. Data Tables 59, 185 (1995).

[31] S. Raman, C. H. Malarkey, W. T. Milner, C. W. Nestor, and D. H. Stelson, At. Data Nucl. Data Tables 36, 1 (1987). 